\title{
THE LIPMAN-ZARISKI CONJECTURE IN GENUS ONE HIGHER
}

\author{
HANNAH BERGNER $^{1}$ and PATRICK GRAF ${ }^{2}$ \\ ${ }^{1}$ Mathematisches Institut, Albert-Ludwigs-Universität Freiburg, Ernst-Zermelo-Straße 1, 79104 \\ Freiburg im Breisgau, Germany; \\ email: Hannah.Bergner-c9q@ruhr-uni-bochum.de \\ ${ }^{2}$ Department of Mathematics, University of Utah, 155 South 1400 East, \\ Salt Lake City, UT 84112, USA; \\ email: patrick.graf@uni-bayreuth.de
}

Received 18 November 2019; accepted 3 March 2020

\begin{abstract}
We prove the Lipman-Zariski conjecture for complex surface singularities with $p_{g}-g-b \leqslant 2$. Here $p_{g}$ is the geometric genus, $g$ is the sum of the genera of exceptional curves and $b$ is the first Betti number of the dual graph. This improves on a previous result of the second author. As an application, we show that a compact complex surface with a locally free tangent sheaf is smooth as soon as it admits two generically linearly independent twisted vector fields and its canonical sheaf has at most two global sections.
\end{abstract}

2010 Mathematics Subject Classification: 14B05 (primary); 14J17, 32S25, 13N15 (secondary)

\section{Introduction}

The Lipman-Zariski conjecture asserts that a complex algebraic variety (or complex space) $X$ with a locally free tangent sheaf $\mathscr{T}_{X}$ is necessarily smooth. Here $\mathscr{T}_{X}=\mathscr{H}_{0 m_{\mathscr{O}_{X}}}\left(\Omega_{X}^{1}, \mathscr{O}_{X}\right)$ is the dual of the sheaf of Kähler differentials. By the combined work of Lipman [Lip65, Theorem 3], Becker [Bec78, Section 8, page 519] and Flenner [Fle88, Corollary], it is known that it suffices to prove the conjecture for normal surface singularities.

In a previous paper [Gra19], the second author dealt with surface singularities that are 'not too far' from being rational. To make this precise, recall that for a normal surface singularity $(X, 0)$, the following invariants are defined in terms of

(C) The Author(s) 2020. This is an Open Access article, distributed under the terms of the Creative Commons Attribution licence (http://creativecommons.org/licenses/by/4.0/), which permits unrestricted re-use, distribution, and reproduction in any medium, provided the original work is properly cited. 
(but not dependent on the choice of) a $\log$ resolution $f: Y \rightarrow X$ with exceptional divisor $E=E_{1}+\cdots+E_{r}$ and dual graph $\Delta=\Delta(E)$ :

$$
\begin{aligned}
p_{g} & :=\operatorname{dim}_{\mathbb{C}}\left(R^{1} f_{*} \mathscr{O}_{Y}\right)_{0}, \text { the (geometric) genus, } \\
g & :=\sum_{i=1}^{r} h^{1}\left(E_{i}, \mathscr{O}_{E_{i}}\right), \\
b & :=b_{1}(\Delta), \quad \text { the first Betti number of } \Delta .
\end{aligned}
$$

In this notation, the main result of [Gra19] (albeit formulated in a different way) is the confirmation of the Lipman-Zariski conjecture in the case $p_{g}-g-b \leqslant 1$. The purpose of this note is to push that result one step further, to $p_{g}-g-b \leqslant 2$. This also explains the title, which on its own is rather cryptic.

THEOREM 1 (Lipman-Zariski conjecture in genus one higher). Let $(X, 0)$ be a normal complex surface singularity, with invariants $p_{g}, g$ and $b$ as above. Assume that $p_{g}-g-b \leqslant 2$. Then the Lipman-Zariski conjecture holds for $(X, 0)$. That is, if $\mathscr{T}_{X}$ is free, then $(X, 0)$ is smooth.

Global Corollaries. In [Gra19], the second author used his (local) main result to study compact complex surfaces whose tangent sheaf satisfies some global triviality properties. Naturally, our stronger Theorem 1 also has new applications in this global setting. First of all, the proof of [Gra19, Corollary 1.4] can be simplified to some extent. For the reader's convenience, we repeat the statement here.

COROLlary 1 (Surfaces with generically nef tangent sheaf). Let $X$ be a complex-projective surface such that $\mathscr{T}_{X}$ is locally free and generically nef. Then $X$ is smooth.

Recall that generic nefness of a vector bundle $\mathscr{E}$ on a normal-projective surface $X$ means the following: there exists an ample line bundle $H$ on $X$ such that if $C \subset X$ is a general element of the linear system $|m H|$, for $m \gg 0$, then the restriction $\left.\mathscr{E}\right|_{C}$ is nef.

A second application concerns compact complex surfaces $X$ that are not necessarily Kähler. By a twisted vector field on $X$, we mean a global section of $\mathscr{T}_{X} \otimes \mathscr{L}$, where $\mathscr{L}$ is a line bundle with vanishing real first Chern class $\mathrm{c}_{1}(\mathscr{L}) \in \mathrm{H}^{2}(X, \mathbb{R})$.

COROLlaRY 2 (Surfaces with two twisted vector fields). Let $X$ be a compact complex surface such that $\mathscr{T}_{X}$ is locally free. Suppose that $X$ admits two twisted 
vector fields $v_{i} \in \mathrm{H}^{0}\left(X, \mathscr{T}_{X} \otimes \mathscr{L}_{i}\right), i=1,2$, which are linearly independent at some point. Assume furthermore that $\operatorname{dim}_{\mathbb{C}} \mathrm{H}^{0}\left(X, \omega_{X}\right) \leqslant 2$. Then $X$ is smooth.

This result generalizes [Gra19, Corollary 1.2], where $X$ was assumed to be almost homogeneous. Note that this is nothing but the special case where both $\mathscr{L}_{i} \cong \mathscr{O}_{X}$.

REMARK 1. The wedge product $v_{1} \wedge v_{2}$ is a nonzero global section of $\omega_{X}^{\vee} \otimes \mathscr{L}_{1} \otimes$ $\mathscr{L}_{2}$, multiplication by which gives an injection $\mathrm{H}^{0}\left(X, \omega_{X}\right) \hookrightarrow \mathrm{H}^{0}\left(X, \mathscr{L}_{1} \otimes \mathscr{L}_{2}\right)$. Thus the assumption on the dimension of $\mathrm{H}^{0}\left(X, \omega_{X}\right)$ is automatically satisfied, for example, if $X$ is Kähler or if $\mathscr{L}_{1} \otimes \mathscr{L}_{2} \cong \mathscr{O}_{X}$.

However, on a non-Kähler surface, having vanishing first Chern class is a rather weak condition on a line bundle. Indeed, a line bundle with $\mathrm{c}_{1}=0$ can have Kodaira dimension one (and hence arbitrarily many global sections). The easiest example is probably given by a Hopf surface of algebraic dimension one. A more interesting example would be a primary Kodaira surface, or more generally any elliptic fibre bundle $S \rightarrow C$ that is not topologically trivial. In this case, $\mathrm{H}^{2}(S, \mathbb{R})$ can be arbitrarily large (depending on $C$ ), but $\varphi^{*}: \mathrm{H}^{2}(C, \mathbb{R}) \rightarrow \mathrm{H}^{2}(S, \mathbb{R})$ always is the zero map [BHPV04, Proposition V.5.3].

REMARK 2. The proof of Corollary 2 shows the following: Assume that for some integer $C$, we knew the Lipman-Zariski conjecture for surface singularities satisfying $p_{g}-g-b \leqslant C$. Then the additional assumption in Corollary 2 can be weakened to " $\operatorname{dim}_{\mathbb{C}} \mathrm{H}^{0}\left(X, \omega_{X}\right) \leqslant C$ ".

\section{Notation and basic facts}

The sheaf of Kähler differentials of a reduced complex space $X$ is denoted by $\Omega_{X}^{1}$. The tangent sheaf, its dual, is denoted by $\mathscr{T}_{X}:=\mathscr{H}$ om $\left(\Omega_{X}^{1}, \mathscr{O}_{X}\right)$. If $Z \subset X$ is a closed subset, then $\mathscr{T}_{X}(-\log Z) \subset \mathscr{T}_{X}$ denotes the subsheaf of vector fields tangent to $Z$ at every point of $Z$. The canonical sheaf of $X$ is denoted by $\omega_{X}$. If $X$ is normal, the sheaf of reflexive differential 1-forms is defined to be the double dual of $\Omega_{X}^{1}$, or the dual of $\mathscr{T}_{X}$. We denote it by $\Omega_{X}^{[1]}:=\left(\Omega_{X}^{1}\right)^{\vee}$. It is isomorphic to $i_{*}\left(\Omega_{X^{\circ}}^{1}\right)$, where $i: X^{\circ} \hookrightarrow X$ is the inclusion of the smooth locus. If $X$ is compact and $\mathscr{F}$ is a coherent sheaf on $X$, we write $h^{i}(X, \mathscr{F}):=\operatorname{dim}_{\mathbb{C}} \mathrm{H}^{i}(X, \mathscr{F})$.

DEFINITION 1 (Resolutions). A resolution of singularities of a reduced complex space $X$ is a proper bimeromorphic morphism $f: Y \rightarrow X$, where $Y$ is smooth. We say that the resolution is projective if $f$ is a projective morphism. A log resolution is a resolution whose exceptional locus $E=\operatorname{Exc}(f)$ is a simple normal crossings 
divisor, that is, a normal crossings divisor with smooth components. A resolution is said to be strong if it is an isomorphism over the smooth locus of $X$.

FACT 1 (Functorial resolutions). Let $X$ be a normal complex space. Then there exists a strong log resolution $f: Y \rightarrow X$ projective over compact subsets, called the functorial resolution, such that $f_{*} \mathscr{T}_{Y}(-\log E)$ is reflexive. This means that for any vector field $\xi \in \Gamma\left(U, \mathscr{T}_{X}\right), U \subset X$ open, there is a unique vector field

$$
\widetilde{\xi} \in \Gamma\left(f^{-1}(U), \mathscr{T}_{Y}(-\log E)\right)
$$

which agrees with $\xi$ wherever $f$ is an isomorphism.

Fact 1 is proven in [Kol07, Theorems 3.36 and 3.45], but concerning the reflexivity of $f_{*} \mathscr{T}_{Y}(-\log E)$ see also [GK14, Theorem 4.2]. If $X$ is a surface, the functorial resolution is the same as the minimal good resolution.

DEFINITION 2 (Geometric genus). Let $(X, 0)$ be a normal surface singularity, and let $f: Y \rightarrow X$ be an arbitrary resolution. The (geometric) genus $p_{g}(X, 0)$ is defined to be the dimension of the stalk $\left(R^{1} f_{*} \mathscr{O}_{Y}\right)_{0}$. Alternatively, choosing the representative $X$ of the germ $(X, 0)$ to be Stein, we may set $p_{g}(X, 0):=$ $\operatorname{dim}_{\mathbb{C}} \mathrm{H}^{1}\left(Y, \mathscr{O}_{Y}\right)$. This definition is independent of the choice of $f$.

The following statement can be found in [Sei67, Theorem 5], in slightly greater generality and with an algebraic proof. Another reference is [BW74, proof of Proposition 1.2]. We include our own proof, which is more geometric in spirit.

Proposition 1 (Derivations in the presence of an isolated singularity). Let $(X, 0)$ be a normal isolated singularity which is not smooth. Then every $\mathbb{C}$-linear derivation $\delta: \mathscr{O}_{X, 0} \rightarrow \mathscr{O}_{X, 0}$ factors through the maximal ideal $\mathfrak{m}_{0} \subset \mathscr{O}_{X, 0}$. In other words, $\delta\left(\mathscr{O}_{X, 0}\right) \subset \mathfrak{m}_{0}$.

In geometric terms, this says that 'every vector field vanishes at the singular point' or more generally, 'every vector field is tangent to the singular locus'.

Proof of Proposition 1. We use the correspondence between derivations, vector fields and local $\mathbb{C}$-actions as described in [Akh95, Sections 1.4, 1.5]. Let $\delta$ be a derivation of $\mathscr{O}_{X, 0}$. We have an induced local $\mathbb{C}$-action $\Phi: \mathbb{C} \times X \rightarrow X$. By the definition of local group action, $\Phi(t,-)$ is an automorphism of the germ $(X, 0)$ for every (sufficiently small) $t \in \mathbb{C}$. Since $0 \in X$ is the unique singular point of $X$, it follows that $\Phi(t, 0)=0$ for every $t \in \mathbb{C}$. In other words, the singular point is fixed by the action $\Phi$. Now, we can recover $\delta$ from $\Phi$ by the formula 


$$
\delta(f)(x)=\left.\frac{\mathrm{d}}{\mathrm{d} t}\right|_{t=0} f(\Phi(t, x))
$$

for every $f \in \mathscr{O}_{X, 0}$. Plugging the statement about the singular point being fixed into (2.2), we arrive at $\delta(f)(0)=0$ for every function germ $f$. Hence $\delta\left(\mathscr{O}_{X, 0}\right) \subset$ $\mathfrak{m}_{0}$, as desired.

Finally, we rely crucially on the following Hodge-theoretic result by van Straten and Steenbrink.

FACT 2 [vSS85, Corollary 1.4]. Let $(X, 0)$ be a normal surface singularity and $f: Y \rightarrow X$ a log resolution with reduced exceptional divisor $E \subset Y$. Then the map

$$
\Omega_{X}^{[1]} / f_{*} \Omega_{Y}^{1} \stackrel{\mathrm{d}}{\longrightarrow} \omega_{X} / f_{*} \omega_{Y}(E)
$$

induced by exterior derivative is injective.

\section{Proof of Theorem 1}

Let $\left\{v_{1}, v_{2}\right\}$ be a local basis of $\mathscr{T}_{X}$ and let $\left\{\alpha_{1}, \alpha_{2}\right\}$ be the dual basis of $\Omega_{X}^{[1]}$, defined by $\alpha_{i}\left(v_{j}\right)=\delta_{i j}$. Furthermore, let $f: Y \rightarrow X$ be the functorial resolution of $X$ and $E \subset Y$ its exceptional locus. We isolate the following observation from the proof of [Gra19, Theorem 1.1], to which we also refer for more details.

OBSERVATION 1. If the basis $\left\{\alpha_{1}, \alpha_{2}\right\}$ can be chosen in such a way that say $\mathrm{d} \alpha_{2} \in$ $f_{*} \omega_{Y}(E)$, that is, $f^{*}\left(\mathrm{~d} \alpha_{2}\right)$ has at most simple poles along $E$, then $(X, 0)$ is smooth.

Sketch of proof. By Fact 2, we see that $\alpha_{2} \in f_{*} \Omega_{Y}^{1}$, that is, $f^{*} \alpha_{2}$ extends to a holomorphic 1-form $\widetilde{\alpha}_{2}$ on $Y$. On the other hand, $v_{2}$ extends to a holomorphic vector field $\widetilde{v}_{2}$ on $Y$ tangent to $E$, by Fact 1 . As $\widetilde{\alpha}_{2}\left(\widetilde{v}_{2}\right)$ is identically one, $\widetilde{v}_{2}$ cannot have any zeros. It follows that $E$, if nonempty, consists of a single smooth elliptic curve. Hence $(X, 0)$ is log canonical and we may apply [GK14, Corollary 1.3]. (We could also appeal to the argument in [vSS85, (1.6)], or in fact even do this case completely by hand.)

CLAIM 1. We have $\operatorname{dim} \omega_{X} / f_{*} \omega_{Y}(E)=p_{g}-g-b$.

Proof. Consider the short exact sequence

$$
0 \longrightarrow \underbrace{f_{*} \omega_{Y}(E) / f_{*} \omega_{Y}}_{=: \mathscr{K}} \longrightarrow \omega_{X} / f_{*} \omega_{Y} \longrightarrow \omega_{X} / f_{*} \omega_{Y}(E) \longrightarrow 0 .
$$


The middle term has dimension exactly $p_{g}$ by [KM98, Proposition 4.45(6)]. Hence it suffices to show that $\operatorname{dim} \mathscr{K}=g+b$. To this end, consider the residue sequence

$$
0 \longrightarrow \omega_{Y} \longrightarrow \omega_{Y}(E) \longrightarrow \omega_{E} \longrightarrow 0
$$

Since $R^{1} f_{*} \omega_{Y}=0$ by Grauert-Riemenschneider vanishing [Kol07, Theorem 2.20.1], and since $E$ is Cohen-Macaulay, we get $\operatorname{dim} \mathscr{K}=h^{0}\left(E, \omega_{E}\right)=$ $h^{1}\left(E, \mathscr{O}_{E}\right)$. A standard computation on the normalization of $E$ yields

$$
h^{1}\left(E, \mathscr{O}_{E}\right)=g+\left|E_{\mathrm{sg}}\right|-r+1 \text {. }
$$

In terms of the dual graph $\Delta=\Delta(E)$, clearly $r$ is the number of vertices and $\left|E_{\mathrm{sg}}\right|$ is the number of edges. But it is a general fact that the first Betti number of a (connected, undirected) graph $G$ with $r$ vertices and $n$ edges is $n-r+1$. (Proof: Let $T \subset G$ be a maximal subtree. Then $T$ has exactly $r-1$ edges. The map $G \rightarrow G / T$ is a homotopy equivalence, and $G / T$ is a wedge sum of $n-(r-1)$ circles.) So $h^{1}\left(E, \mathscr{O}_{E}\right)=g+b$, as desired.

Claim 2. The 2-form $\sigma:=\alpha_{1} \wedge \alpha_{2}$ is a generator of $\omega_{X}$. In particular, $X$ is Gorenstein.

Proof. Define a map $\mathscr{O}_{X} \rightarrow \omega_{X}$ by sending $1 \mapsto \sigma$. This is an isomorphism on the smooth locus $X \backslash\{0\}$. Then it is an isomorphism everywhere, as $X$ is normal and the sheaves $\mathscr{O}_{X}$ and $\omega_{X}$ are reflexive.

By Claim 2, every element in $\omega_{X} / f_{*} \omega_{Y}(E)$ can be written as (the class of) $\rho \cdot \sigma$ for some holomorphic function germ $\rho \in \mathscr{O}_{X, 0}$. If $\mathfrak{m}:=\mathfrak{m}_{0} \subset \mathscr{O}_{X, 0}$ is the maximal ideal, consider the linear subspace

$$
\mathfrak{m} \omega_{X} / f_{*} \omega_{Y}(E)=\{\rho \cdot \sigma \mid \rho(0)=0\} \subset \omega_{X} / f_{*} \omega_{Y}(E)
$$

Unless $\omega_{X} / f_{*} \omega_{Y}(E)=0$, this subspace has codimension one. In any case, it has dimension $\leqslant 1$ by Claim 1 and the assumption $p_{g}-g-b \leqslant 2$. (This is the only place where that assumption is used.) Thus if the images of $\mathrm{d} \alpha_{1}$ and $\mathrm{d} \alpha_{2}$ are both contained in $\mathfrak{m} \omega_{X} / f_{*} \omega_{Y}(E)$, they are linearly dependent, say $\mathrm{d} \alpha_{1}+\lambda \cdot \mathrm{d} \alpha_{2}=$ 0 for some $\lambda \in \mathbb{C}$. Considering the basis $\left\{\alpha_{1}+\lambda \alpha_{2}, \alpha_{2}\right\}$ of $\Omega_{X}^{[1]}$, we can apply Observation 1 to conclude that $(X, 0)$ is smooth. After possibly interchanging $\alpha_{1}$ and $\alpha_{2}$, we may hence without loss of generality make the following

AdDitional Assumption 1. We have $\mathrm{d} \alpha_{1} \notin \mathfrak{m} \omega_{X}$. 
Writing $\mathrm{d} \alpha_{j}=\rho_{j} \cdot \sigma$ for suitable $\rho_{j} \in \mathscr{O}_{X, 0}$, we thus have that $\rho_{1} \notin \mathfrak{m}$ is a unit. So replacing $\alpha_{2}$ by $\rho_{1} \alpha_{2}$ does not destroy the property of $\left\{\alpha_{1}, \alpha_{2}\right\}$ being a basis of $\Omega_{X}^{[1]}$. After this replacement, $\mathrm{d} \alpha_{1}=\sigma$. Furthermore, note that

$$
\mathrm{d}(\underbrace{\alpha_{2}-\rho_{2}(0) \alpha_{1}}_{=: \alpha_{2}^{\prime}})=(\underbrace{\rho_{2}-\rho_{2}(0)}_{\in \mathfrak{m}}) \cdot \sigma,
$$

and that we may replace $\alpha_{2}$ by $\alpha_{2}^{\prime}$, again without destroying the basis property. Summing up, this leads to the following simplification of our setting.

Additional Assumption 2. We have that $\mathrm{d} \alpha_{1}=\sigma$ and $\mathrm{d} \alpha_{2} \in \mathfrak{m} \omega_{X}$. In other words, $\rho_{1} \equiv 1$ and $\rho_{2} \in \mathfrak{m}$.

We will also assume from now on that $(X, 0)$ is not smooth, as otherwise there is nothing to prove. Consider the 1-form $\rho_{2} \alpha_{1}$. A short calculation shows that $\mathrm{d}\left(\rho_{2} \alpha_{1}\right)=\left(\rho_{2}-v_{2}\left(\rho_{2}\right)\right) \cdot \sigma$, which by Proposition 1 and Assumption 2 defines an element in the at most one-dimensional vector space $\mathfrak{m} \omega_{X} / f_{*} \omega_{Y}(E)$. If that element is nonzero, then there is a constant $\lambda \in \mathbb{C}$ with

$$
\mathrm{d}\left(\alpha_{2}+\lambda \rho_{2} \alpha_{1}\right)=\mathrm{d} \alpha_{2}+\lambda \mathrm{d}\left(\rho_{2} \alpha_{1}\right)=0 \in \mathfrak{m} \omega_{X} / f_{*} \omega_{Y}(E) \subset \omega_{X} / f_{*} \omega_{Y}(E) .
$$

Because $\left\{\alpha_{1}, \alpha_{2}+\lambda \rho_{2} \alpha_{1}\right\}$ is a basis of $\Omega_{X}^{[1]}$, we can again apply Observation 1 and then we are done. Hence we may without loss of generality impose the following

Additional Assumption 3. We have d $\left(\rho_{2} \alpha_{1}\right) \in f_{*} \omega_{Y}(E)$.

For any function/differential form/vector field on $X$, we denote its lift to $Y$ as a holomorphic or meromorphic object by a tilde. Thus we have, for example, $\mathrm{d} \widetilde{\alpha}_{1}=\widetilde{\sigma}=\widetilde{\alpha}_{1} \wedge \widetilde{\alpha}_{2}$ and $\mathrm{d} \widetilde{\alpha}_{2}=\widetilde{\rho}_{2} \cdot \widetilde{\sigma}$. Furthermore we let $\mathfrak{E}$ be the set of irreducible components of $E$ and we put

$$
\begin{aligned}
& \mathfrak{E}^{\leqslant 1}:=\left\{P \in \mathfrak{E} \mid \widetilde{v}_{2} \text { vanishes to order at most } 1 \text { along } P\right\}, \\
& \mathfrak{E} \geqslant 2:=\left\{P \in \mathfrak{E} \mid \widetilde{v}_{2} \text { vanishes to order at least } 2 \text { along } P\right\} .
\end{aligned}
$$

The order of vanishing, of course, refers to the largest integer $k$ such that locally near a general point of $P$, we can write $\widetilde{v}_{2}=w^{k} v^{\prime}$, where $w$ is a local defining equation for $P$ and $v^{\prime}$ is a holomorphic vector field.

Let $P \in \mathfrak{E}^{\geqslant 2}$ be arbitrary, pick a point $p \in P$ not contained in any other component of $E$, and choose local holomorphic coordinates $z, w$ around $p$ such that locally $P=\{w=0\}$. We can then write $\widetilde{v}_{2}=w^{2} v^{\prime}$ for some holomorphic vector field $v^{\prime}$ defined near $p$. The 1 -form $\alpha_{2}-\rho_{2} \alpha_{1}$ satisfies

$$
\mathrm{d}\left(\alpha_{2}-\rho_{2} \alpha_{1}\right)=\left[\rho_{2}-\left(\rho_{2}-v_{2}\left(\rho_{2}\right)\right)\right] \cdot \sigma=v_{2}\left(\rho_{2}\right) \cdot \sigma,
$$


and the order of vanishing of $f^{*}\left(v_{2}\left(\rho_{2}\right)\right)=\widetilde{v}_{2}\left(\widetilde{\rho}_{2}\right)=w^{2} v^{\prime}\left(\widetilde{\rho}_{2}\right)$ along $P$ is strictly larger than the vanishing order of $\tilde{\rho}_{2}$ along $P$. Hence after replacing $\alpha_{2}$ by $\alpha_{2}-$ $\rho_{2} \alpha_{1}$ finitely often, the 2 -form $\mathrm{d} \widetilde{\alpha}_{2}=\widetilde{\rho}_{2} \cdot \widetilde{\sigma}$ will be holomorphic at a general point of $P$. This argument applies simultaneously to all $P \in \mathfrak{E} \geqslant 2$ and we arrive at the

Additional Assumption 4. The 2-form d $\widetilde{\alpha}_{2}$ does not have a pole along any exceptional curve $P \in \mathfrak{E}^{\geqslant 2}$.

The next claim analogously deals with $\mathfrak{E} \leqslant 1$. We stress that its proof relies only on Assumption 3, but not on Assumption 4.

Claim 3. Along any curve $P \in \mathfrak{E}^{\leqslant 1}$, the form $\mathrm{d} \widetilde{\alpha}_{2}$ has at worst a simple pole.

Proof of Claim 3. Pick a component $P \in \mathfrak{E}^{\leqslant 1}$ and let $p \in P$ and $z, w$ be as before. There are holomorphic functions $a$ and $b$ near $p$ such that locally $\widetilde{v}_{2}=a \frac{\partial}{\partial z}+b \frac{\partial}{\partial w}$. Using Taylor expansion, we may write

$$
\begin{aligned}
& a(z, w)=a_{0}(z)+a_{1}(z) w+\cdots \quad \text { and } \\
& b(z, w)=b_{0}(z)+b_{1}(z) w+\cdots,
\end{aligned}
$$

where the dots stand for terms of order at least 2 in $w$ and $a_{j}, b_{j}$ are appropriate local holomorphic functions in one variable. Since $\widetilde{v}_{2}$ is logarithmic with respect to $P=\{w=0\}$, we in fact have $b_{0} \equiv 0$. As $P \in \mathfrak{E}^{\leqslant 1}$, not all of $a_{0}, a_{1}, b_{1}$ can be identically zero.

○ If $a_{0} \not \equiv 0$, there is a point $q \in P$ near $p$ with $a_{0}(q) \neq 0$.

○ If $a_{0} \equiv 0$, we may locally write $\widetilde{v}_{2}=w \cdot v^{\prime}$ with $v^{\prime}$ holomorphic. Since $a_{1} \not \equiv 0$ or $b_{1} \not \equiv 0$, there is a point $q \in P$ near $p$ with $v^{\prime}(q) \neq 0$.

In both cases, locally near $q$ we have $\widetilde{v}_{2}=h \cdot v^{\prime}$ for a holomorphic function $h$ and a holomorphic vector field $v^{\prime}$ with $v^{\prime}(q) \neq 0$. What is more, the function $h$ (which is either identically one, or equal to $w$ ) vanishes of order at most one along $P$. Since $v^{\prime}(q) \neq 0$, there exist local holomorphic coordinates $x, y$ near $q$ such that locally $v^{\prime}=\frac{\partial}{\partial x}$ and thus $\widetilde{v}_{2}=h \cdot \frac{\partial}{\partial x}$.

There are local meromorphic functions $g_{i j}$ such that with respect to the local coordinates $x, y$ we have $\widetilde{\alpha}_{1}=g_{11} \mathrm{~d} x+g_{12} \mathrm{~d} y$ and $\widetilde{\alpha}_{2}=g_{21} \mathrm{~d} x+g_{22} \mathrm{~d} y$. Because $\widetilde{\alpha}_{i}\left(\widetilde{v}_{2}\right)=\delta_{i, 2}$, we have in fact $\widetilde{\alpha}_{1}=g_{12} \mathrm{~d} y$ and $\widetilde{\alpha}_{2}=h^{-1} \mathrm{~d} x+g_{22} \mathrm{~d} y$. Thus by Assumption 2

$$
\mathrm{d} \widetilde{\alpha}_{1}=\widetilde{\sigma}=\widetilde{\alpha}_{1} \wedge \widetilde{\alpha}_{2}=-\frac{g_{12}}{h} \mathrm{~d} x \wedge \mathrm{d} y
$$


and

$$
\mathrm{d} \widetilde{\alpha}_{2}=\widetilde{\rho}_{2} \cdot \widetilde{\sigma}=-\frac{\tilde{\rho}_{2} g_{12}}{h} \mathrm{~d} x \wedge \mathrm{d} y
$$

According to Assumption 3 and Fact 2, $f^{*}\left(\rho_{2} \alpha_{1}\right)=\widetilde{\rho}_{2} \widetilde{\alpha}_{1}=\widetilde{\rho}_{2} g_{12} \mathrm{~d} y$ extends to a holomorphic 1-form on $Y$. In particular, $\widetilde{\rho}_{2} g_{12} \mathrm{~d} y$ has no pole along $P$ and therefore $\widetilde{\rho}_{2} g_{12}$ is holomorphic. This implies that $\mathrm{d} \widetilde{\alpha}_{2}=-h^{-1} \widetilde{\rho}_{2} g_{12} \mathrm{~d} x \wedge \mathrm{d} y$ has at most a pole of order one along $P$, as desired.

Taken together, Assumption 4 and Claim 3 show that $\mathrm{d} \widetilde{\alpha}_{2}$ has at worst firstorder poles along any exceptional curve $P \in \mathfrak{E}=\mathfrak{E}^{\leqslant 1} \cup \mathfrak{E}^{\geqslant 2}$. In other words, we have $\mathrm{d} \alpha_{2} \in f_{*} \omega_{Y}(E)$. Applying once again Observation 1 , we get that $(X, 0)$ is smooth.

\section{Proof of Corollary 1}

By [Lip65, Theorem 3], $X$ is normal. Let $f: S \rightarrow X$ be the minimal resolution (that is, $K_{S}$ is $f$-nef). We may assume that $X$ is not smooth. Under this additional assumption, one shows as in the proof of [Gra19, Claim 4.2] that

$$
h^{0}\left(X, R^{1} f_{*} \mathscr{O}_{S}\right)=\sum_{x \in X_{\mathrm{sg}}} p_{g}(X, x) \leqslant 2 .
$$

Hence every singular point of $X$ satisfies $p_{g}-g-b \leqslant p_{g} \leqslant 2$. We conclude by Theorem 1 that $X$ is smooth.

\section{Proof of Corollary 2}

The following proposition, probably well known to experts, will greatly simplify the proof.

PROPOSITION 2 (Surfaces carrying a divisor homologous to zero). Let $S$ be a smooth compact complex surface containing a nonzero effective divisor $D$ with

$$
\mathrm{c}_{1}(D):=\mathrm{c}_{1}\left(\mathscr{O}_{S}(D)\right)=0 \in \mathrm{H}^{2}(S, \mathbb{R}) .
$$

Then either

(2.1) the Kodaira dimension $\kappa(S) \leqslant 0$; or

(2.2) we have $\kappa(S)=1$ and $\chi\left(\mathscr{O}_{S}\right)=0$. 
Proof. Let $\pi: S \rightarrow S_{0}$ be a minimal model, and set $D_{0}:=\pi_{*} D$. Then $\mathrm{c}_{1}\left(D_{0}\right)=0$ by Lemma 1 below. Furthermore $D_{0} \neq 0$, as otherwise $D$ would be $\pi$-exceptional and hence $D^{2}<0$ by negative definiteness of the intersection form [BHPV04, Theorem III.2.1], contradicting the fact that $D^{2}=\mathrm{c}_{1}(D)^{2}=0$. Also $\chi\left(\mathscr{O}_{S}\right)$ remains unchanged when passing to $S_{0}$. We may thus assume that $S$ is minimal.

If $\kappa(S)=2$, then $\mathrm{c}_{1}^{2}(S)>0$ and $S$ is projective [BHPV04, Theorem IV.6.2]. Thus the divisor $D$ cannot exist. If $\kappa(S)=1$, then the pluricanonical map $\varphi: S \rightarrow$ $C$ is a relatively minimal elliptic fibration. In this case

$$
\begin{aligned}
\chi\left(\mathscr{O}_{S}\right) & =\operatorname{deg}_{C}\left(R^{1} \varphi_{*} \mathscr{O}_{S}\right)^{\vee} & & \text { [BHPV04, Proposition V.12.2] } \\
& =\operatorname{deg}_{C}\left(\varphi_{*} \omega_{S / C}\right) & & {[\text { BHPV04, Theorem III.12.3] }} \\
& \geqslant 0 . & & \text { [BHPV04, Theorem III.18.2] }
\end{aligned}
$$

On the other hand, we have $\chi\left(\mathscr{O}_{S}\right) \leqslant 0$ by [CDP98, Corollary 1.2]. We conclude that $\chi\left(\mathscr{O}_{S}\right)=0$.

LEMMA 1. Let $S$ be a smooth compact complex surface and $\pi: S \rightarrow S^{\prime}$ the blowing-down of a $(-1)$-curve. If $\mathscr{L} \in \operatorname{Pic}(S)$ is a line bundle with $\mathrm{c}_{1}(\mathscr{L})=0$, then so is $\mathscr{L}^{\prime}:=\left(\pi_{*} \mathscr{L}\right)^{\vee}$, where $(-)^{\vee}$ denotes the reflexive hull (or double dual) of a coherent sheaf.

Proof. Being a reflexive rank-1 sheaf on a smooth surface, $\mathscr{L}^{\prime}$ is locally free. Thanks to negative definiteness again [BHPV04, Theorem III.2.1], we have $\mathscr{L}=$ $\pi^{*} \mathscr{L}^{\prime}$ and hence $\pi^{*}\left(\mathrm{c}_{1}\left(\mathscr{L}^{\prime}\right)\right)=\mathrm{c}_{1}(\mathscr{L})=0$. As $\pi^{*}: \mathrm{H}^{2}\left(S^{\prime}, \mathbb{R}\right) \rightarrow \mathrm{H}^{2}(S, \mathbb{R})$ is injective [BHPV04, Theorem I.9.1(iv)], it follows that $\mathrm{c}_{1}\left(\mathscr{L}^{\prime}\right)=0$.

LEMMA 2. Let $\mathscr{L}$ be a line bundle on the smooth-projective curve $C$ of genus $g$.

(2.3) If $\operatorname{deg} \mathscr{L}=0$, then $h^{0}(C, \mathscr{L})=h^{0}\left(C, \mathscr{L}^{\vee}\right)$.

(2.4) If $\operatorname{deg} \mathscr{L}=2 g-2$ but $\mathscr{L}$ is not isomorphic to $\omega_{C}$, then $h^{0}(C, \mathscr{L})=g-1$.

Proof. This is well known and hence left to the reader as an exercise.

We now turn to the proof of Corollary 2. As in the previous corollary, we may assume that $X$ is normal, but not smooth. Let $f: S \rightarrow X$ be the functorial resolution and $\pi: S \rightarrow S_{0}$ a run of the $K_{S}$-MMP.

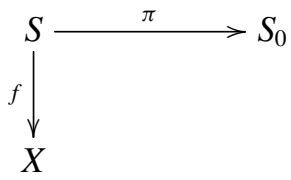


By Fact 1 , the twisted vector fields $v_{i}$ on $X$ lift to twisted vector fields $\widetilde{v}_{i}$ on $S$. These in turn can be pushed forward to twisted vector fields $v_{i}^{0}$ on $S_{0}$, by Lemma 1 . Furthermore, the Leray spectral sequence associated to $f_{*} \mathscr{O}_{S}$ yields a five-term exact sequence

$$
\begin{aligned}
0 & \longrightarrow \mathrm{H}^{1}\left(X, \mathscr{O}_{X}\right) \longrightarrow \mathrm{H}^{1}\left(S, \mathscr{O}_{S}\right) \longrightarrow \mathrm{H}^{0}\left(X, R^{1} f_{*} \mathscr{O}_{S}\right) \\
& \longrightarrow \mathrm{H}^{2}\left(X, \mathscr{O}_{X}\right) \longrightarrow \mathrm{H}^{2}\left(S, \mathscr{O}_{S}\right) \longrightarrow 0,
\end{aligned}
$$

where the last map is Serre dual to $\mathrm{H}^{0}\left(S, \omega_{S}\right) \hookrightarrow \mathrm{H}^{0}\left(X, \omega_{X}\right)$, and hence surjective. We obtain an upper bound

$$
h^{0}\left(X, R^{1} f_{*} \mathscr{O}_{S}\right) \leqslant h^{1}\left(S, \mathscr{O}_{S}\right)+h^{0}\left(X, \omega_{X}\right)-h^{0}\left(S, \omega_{S}\right) .
$$

Claim 4. Assume that $S$ has the property that every nonzero effective divisor $D \subset S$ satisfies $\mathrm{c}_{1}(D) \neq 0$. (This applies in particular if $S$ is Kähler.) Then $h^{0}\left(X, \omega_{X}\right) \leqslant 1$ and $\kappa(S)=-\infty$.

Proof. If $\kappa\left(X, K_{X}\right)=-\infty$, then in particular $h^{0}\left(X, \omega_{X}\right)=0$ and also $\kappa(S)=$ $-\infty$, since in any case $\kappa(S) \leqslant \kappa\left(X, K_{X}\right)$. We may thus assume that $\kappa\left(X, K_{X}\right) \geqslant 0$, that is, $\left|m K_{X}\right| \neq \varnothing$ for some $m \geqslant 1$. Pick $D_{m} \in\left|m K_{X}\right|$, for a suitable $m$. The wedge product of twisted vector fields $v_{1} \wedge v_{2}$ is a nonzero global section of $\omega_{X}^{\vee} \otimes \mathscr{L}_{1} \otimes \mathscr{L}_{2}$. Its zero divisor is thus an element $D_{-1} \in\left|-K_{X}+L_{1}+L_{2}\right|$. Then $D_{m}+m D_{-1} \in\left|m\left(L_{1}+L_{2}\right)\right|$ is an effective divisor with first Chern class zero. It follows that $D_{m}+m D_{-1}=0$ (pull back along $f$ and use the assumption on $S$ ). Hence $D_{m}=0$, that is, $K_{X}$ is torsion and $h^{0}\left(X, \omega_{X}\right) \leqslant 1$. If $f^{\prime}: S^{\prime} \rightarrow X$ is the minimal resolution, we have

$$
K_{S^{\prime}}=f^{*} K_{X}-E \sim_{\mathbb{Q}}-E
$$

with $E \geqslant 0$ an effective $f^{\prime}$-exceptional divisor. If $E=0$, then $X$ has canonical singularities; hence it is smooth [GK14, Corollary 1.3]. So $E \geqslant 0$ and $\kappa(S)=$ $\kappa\left(S^{\prime}\right)=\kappa\left(S^{\prime},-E\right)=-\infty$.

Claim 5. If $\varphi: S_{0} \rightarrow C$ is a ruled surface, then the genus $g(C) \leqslant 1$.

Proof. The vector fields $v_{i}^{0}$, being generically linearly independent, cannot both be tangent to the fibres of $\varphi$. Hence $\mathrm{H}^{0}\left(S_{0}, \varphi^{*} \mathscr{T}_{C} \otimes \mathscr{L}_{i}\right) \neq 0$ for, say, $i=1$. This is the same as $\mathrm{H}^{0}\left(C, \mathscr{T}_{C} \otimes \varphi_{*} \mathscr{L}_{1}\right)$ by the projection formula, so $\varphi_{*} \mathscr{L}_{1} \neq 0$. Since $\mathrm{c}_{1}\left(\mathscr{L}_{1}\right)=0, \mathscr{L}_{1}$ must be trivial on the fibres of $\varphi$. Thus $\varphi_{*} \mathscr{L}_{1}$ is a line bundle and $\mathscr{L}_{1}=\varphi^{*}\left(\varphi_{*} \mathscr{L}_{1}\right)$. We conclude from this that $\operatorname{deg}_{C} \varphi_{*} \mathscr{L}_{1}=0$. Summing up, the line bundle $\mathscr{T}_{C} \otimes \varphi_{*} \mathscr{L}_{1}$ has a nonzero global section (the image of $v_{1}$ ) and its 
Table 1. Possibilities for $S_{0}$ and corresponding dimensions of cohomology groups.

\begin{tabular}{llll}
\hline$S_{0}$ & $h^{1}\left(S, \mathscr{O}_{S}\right)$ & $h^{0}\left(X, \omega_{X}\right)$ & $h^{0}\left(S, \omega_{S}\right)$ \\
\hline $\mathbb{P}^{2}$ or ruled & $\leqslant 1$ (Claim 5) & $\leqslant 1$ (Claim 4) & 0 \\
Class VII ${ }_{0}$ & 1 [BHPV04, Theorem IV.2.7] & $\leqslant 2$ & 0 \\
Primary Kodaira & 2 & $\leqslant 2$ & 1 \\
Secondary Kodaira & 1 & $\leqslant 2$ & 0 \\
Minimal properly & $g+h^{0}(L)$ & $\leqslant 2$ & $\geqslant h^{0}\left(K_{C}+L\right)$ \\
elliptic, non-Kähler, & & & \\
and $\chi\left(\mathscr{O}_{S}\right)=0$ & & & \\
\hline
\end{tabular}

degree is $2-2 g(C)+\operatorname{deg}_{C} \varphi_{*} \mathscr{L}_{1}=2-2 g(C) \geqslant 0$. This immediately implies the claim.

Claim 6. If $\kappa(S)=1$ and $\varphi: S_{0} \rightarrow C$ is the pluricanonical map, let $L$ be a divisor on $C$ corresponding to the line bundle $\varphi_{*} \omega_{S_{0} / C}$. Assume that $\operatorname{deg} L=0$. Then

$\circ h^{1}\left(S, \mathscr{O}_{S}\right)=g+h^{0}(C, L)$, where $g$ is the genus of $C$, and

○ $h^{0}\left(S, \omega_{S}\right) \geqslant h^{0}\left(C, K_{C}+L\right)$.

Proof. The Leray spectral sequence for $\varphi$ and $\mathscr{O}_{S_{0}}$ gives

$$
h^{1}\left(S_{0}, \mathscr{O}_{S_{0}}\right)=h^{1}\left(C, \mathscr{O}_{C}\right)+h^{0}\left(C, R^{1} \varphi_{*} \mathscr{O}_{S_{0}}\right)=g+h^{0}(C, L)
$$

by (2.3). On the other hand, if $m_{1} F_{1}, \ldots, m_{k} F_{k}, m_{i} \geqslant 2$, are the multiple fibres of $\varphi$, then Kodaira's canonical bundle formula [BHPV04, Theorem V.12.1] reads

$$
K_{S_{0}}=\varphi^{*}\left(K_{C}+L\right)+\sum_{i=1}^{k}\left(m_{i}-1\right) F_{i} \geqslant \varphi^{*}\left(K_{C}+L\right) .
$$

Taking global sections yields the second claim.

Now by Claim 4, either $\kappa(S)=-\infty$, or $\kappa(S) \in\{0,1\}$ and $S$ contains a divisor with vanishing first Chern class. By Proposition 2 and the Kodaira-Enriques classification [BHPV04, Table 10 on page 244], we are left with the possibilities for $S_{0}$ listed in Table 1 on this page. 
In each case, the estimate (5.3) yields $h^{0}\left(X, R^{1} f_{*} \mathscr{O}_{S}\right) \leqslant h^{0}\left(X, \omega_{X}\right)+1$. In the last case, this is seen as follows, using (2.4):

$$
g+h^{0}(C, L)-h^{0}\left(C, K_{C}+L\right)=\left\{\begin{array}{ll}
g+1-g, & L \text { trivial, } \\
g-(g-1), & L \text { nontrivial },
\end{array}\right\}=1 .
$$

Hence if $h^{0}\left(X, \omega_{X}\right) \leqslant 1$, then we can conclude by Theorem 1 that $X$ is smooth, just as in the proof of Corollary 1. We will thus from now on assume that $h^{0}\left(X, \omega_{X}\right)=2$. In view of the above table, this means that the first line $\left(S_{0}=\mathbb{P}^{2}\right.$ or ruled) can be excluded. Also, the algebraic dimension $\mathrm{a}\left(S_{0}\right)=1$, as the ratio of two linearly independent sections of $\omega_{X}$ provides a nonconstant meromorphic function on $X$ and then also on $S_{0}$.

ClaIM 7. Any irreducible curve contained in $S_{0}$ is smooth elliptic.

Proof. Assume first that $S_{0}$ is a primary Kodaira surface, that is, in particular a locally trivial fibration with fibre $F$ an elliptic curve. If $C \subset S_{0}$ were a curve not contained in a fibre, then $(C+n F)^{2}>0$ for $n \gg 0$ and so $S_{0}$ would be projective [BHPV04, Theorem IV.6.2], which it is not. Hence Claim 7 is true in this case. A secondary Kodaira surface admits an étale covering by a primary Kodaira surface, so any of its curves must be smooth and then also elliptic by the Hurwitz formula.

We next treat the case where $S_{0}$ is of class $\mathrm{VII}_{0}$. By [Kod66, Theorem 35], $S_{0}$ is a Hopf surface. As any Hopf surface has an étale covering by a primary Hopf surface [Kod66, Theorem 30], we may assume by the same argument as above that $S_{0}$ is itself primary. Then $S_{0}$ is the quotient of $W:=\mathbb{C}^{2} \backslash\{0\}$ by the infinite cyclic group $G$ generated by the automorphism $\left(z_{1}, z_{2}\right) \mapsto\left(\alpha_{1} z_{1}, \alpha_{2} z_{2}\right)$, where $0<\left|\alpha_{1}\right| \leqslant\left|\alpha_{2}\right|<1$ and $\alpha_{1}^{k}=\alpha_{2}^{\ell}$ for certain positive integers $k, \ell$ [Kod66, Theorem 31]. We may assume that $k$ and $\ell$ are minimal with this property. The nonconstant meromorphic function $z_{1}^{k} / z_{2}^{\ell}$ then defines a map $\varphi: S_{0} \rightarrow \mathbb{P}^{1}$ with connected fibres. We claim that all fibres of $\varphi$ are smooth elliptic curves. To this end, let $\tilde{\varphi}: W \rightarrow \mathbb{P}^{1}$ be the pullback of $\varphi$ to the universal covering. By calculating the differential of $\tilde{\varphi}$, we see that this map has rank one at all points $\left(z_{1}, z_{2}\right) \in W$ with $z_{1} z_{2} \neq 0$. So all the fibres $F_{\lambda}:=\varphi^{-1}(\lambda)$ with $\lambda \neq 0, \infty$ are smooth. As the second Betti number $b_{2}\left(S_{0}\right)=0$, all intersection numbers on $S_{0}$ are zero. In particular, deg $K_{F_{\lambda}}=\left(K_{S_{0}}+F_{\lambda}\right) \cdot F_{\lambda}=0$ by adjunction and so $F_{\lambda}$ is an elliptic curve.

It remains to consider $F_{0}$ and $F_{\infty}$. The fibre $F_{0}$ is the quotient of $\widetilde{\varphi}^{-1}(0)=$ $\left\{z_{1}=0\right\} \cong \mathbb{C}^{*}$ by the group $G$, which acts on $\widetilde{\varphi}^{-1}(0)$ via multiplication by $\alpha_{2}$. Via the exponential map, $F_{0}$ is thus seen to be isomorphic to $\mathbb{C}$ modulo a lattice, that is, an elliptic curve. The argument for $F_{\infty}$ is the same. So all fibres of $\varphi$ are 
smooth elliptic. As in the case of Kodaira surfaces, every curve on $S_{0}$ is contained in a fibre of $\varphi$ and hence the claim is proven in this case, too.

Finally, if $S_{0}$ is minimally elliptic and $\varphi: S_{0} \rightarrow C$ is the pluricanonical map, then we have seen that $\operatorname{deg}_{C}\left(\varphi_{*} \omega_{S_{0} / C}\right)=0$. By [BHPV04, Theorem III.18.2] this implies that the only singular fibres of $\varphi$ are multiples of smooth elliptic curves. In particular, set-theoretically all fibres are smooth elliptic. Again, there are no other curves except the fibres and so the proof is finished.

As observed above, we have $h^{0}\left(X, R^{1} f_{*} \mathscr{O}_{S}\right) \leqslant 3$. If $X$ has only singularities of genus at most two, we conclude by Theorem 1. Otherwise, there is a genus 3 singularity $x \in X$, and it is the unique singular point of $X$. If $\operatorname{Exc}(f)$ contains a nonrational curve, then $x \in X$ has $g \geqslant 1$; hence $p_{g}-g-b \leqslant 2$, and we are done by Theorem 1 again. If, on the other hand, $\operatorname{Exc}(f)$ consists solely of rational curves, then in particular every $f$-exceptional curve gets contracted to a point by $\pi$ thanks to Claim 7. In other words, $\operatorname{Exc}(f) \subset \operatorname{Exc}(\pi)$. By the Theorem on Formal Functions, $R^{1} f_{*} \mathscr{O}_{S}$ can be computed as

$$
\lim _{Z} H^{1}\left(Z, \mathscr{O}_{Z}\right)
$$

where the inverse limit runs over all cycles $Z$ with $\operatorname{supp} Z \subset \operatorname{Exc}(f)$. By smoothness of $S_{0}$, we have $R^{1} \pi_{*} \mathscr{O}_{S}=0$ and thus, by the Theorem on Formal Functions again, $\mathrm{H}^{1}\left(Z, \mathscr{O}_{Z}\right)=0$ for all $Z$ with $\operatorname{supp} Z \subset \operatorname{Exc}(\pi)$. Since $\operatorname{Exc}(f) \subset \operatorname{Exc}(\pi)$, we conclude that $x \in X$ is a rational singularity and so $X$ is smooth by Theorem 1 .

REMARK. We would like to discuss which parts of the above argument can be generalized, in particular with respect to Table 1 . In the first case, $S_{0}=\mathbb{P}^{2}$ or a ruled surface, the condition $h^{0}\left(X, \omega_{X}\right) \leqslant 2$ is automatic by Claim 4 , but the existence of two twisted vector fields on $X$ is necessary in Claim 5 to exclude ruled surfaces over curves of general type. If there is only one vector field, all one can say is that such a ruled surface would be decomposable.

The vector fields $v_{1,2}$ are also used in Claim 4 to rule out the situation that $S_{0}$ is Kähler and of nonnegative Kodaira dimension. Without this assumption, several new cases need to be dealt with:

○ If $S_{0}$ is a complex 2-torus, then $h^{1}\left(S, \mathscr{O}_{S}\right)-h^{0}\left(S, \omega_{S}\right)=1$ and so we can still prove smoothness of $X$ if $h^{0}\left(X, \omega_{X}\right) \leqslant 2$. Note that Claim 7 does not hold any longer, but this is not a problem because it is still true that $S_{0}$ contains no rational curves.

○ If $S_{0}$ is bi-elliptic, then again $h^{1}\left(S, \mathscr{O}_{S}\right)-h^{0}\left(S, \omega_{S}\right)=1$ and there are no rational curves on $S_{0}$. The conclusion is thus the same as in the torus case. 
○ If $S_{0}$ is a K3 surface, we have $h^{1}\left(S, \mathscr{O}_{S}\right)-h^{0}\left(S, \omega_{S}\right)=-1$. Thus the assumption $h^{0}\left(X, \omega_{X}\right) \leqslant 3$ is sufficient. The case $h^{0}\left(X, \omega_{X}\right)=4$, however, appears difficult due to the failure of Claim 7: $S_{0}$ could certainly contain a lot of rational curves.

○ If $S_{0}$ is an Enriques surface, then $h^{1}\left(S, \mathscr{O}_{S}\right)-h^{0}\left(S, \omega_{S}\right)=0$. Similarly to the $\mathrm{K} 3$ case, $h^{0}\left(X, \omega_{X}\right) \leqslant 2$ is fine, but $h^{0}\left(X, \omega_{X}\right)=3$ is not.

○ If $\kappa\left(S_{0}\right)=1$, we use notation as in Claim 6. If $\operatorname{deg} L=0$, we have already seen that $h^{1}\left(S, \mathscr{O}_{S}\right)-h^{0}\left(S, \omega_{S}\right) \leqslant 1$. If $h^{0}\left(X, \omega_{X}\right) \leqslant 1$ or if $g>0$, the above arguments apply. The remaining case $h^{0}\left(X, \omega_{X}\right)=2$ and $g=0$ needs to be addressed either by showing $h^{0}\left(S, \omega_{S}\right)=1$ or by excluding any rational curves on $S_{0}$.

If $\operatorname{deg} L>0$, then clearly $h^{1}\left(S, \mathscr{O}_{S}\right)=g+h^{0}\left(L^{\vee}\right)=g$ and $h^{0}\left(S, \omega_{S}\right) \geqslant$ $h^{0}\left(K_{C}+L\right) \geqslant g$; hence the difference is $\leqslant 0$. The conclusion is as in the Enriques case because the singular fibres of $\varphi$ can very well have rational components.

o If $S_{0}$ is of general type, it appears difficult (if not impossible) to give a general upper bound on $h^{1}\left(S, \mathscr{O}_{S}\right)-h^{0}\left(S, \omega_{S}\right)$ and hence we do not believe that statements in the style of Theorem 1 are useful for handling this situation.

\section{Acknowledgements}

We would like to thank Stéphane Druel for pointing out to us reference [Sei67]. The second author thanks the University of Utah for providing a most hospitable working environment. We are also grateful to the referee for constructive criticism, in particular for suggesting a reformulation of the main result that is not only more concise but also more general.

Funding information. The second author was supported in full by a Research Fellowship of the German Research Foundation (DFG). This publication was funded by the DFG and the University of Bayreuth in the funding programme Open Access Publishing.

\section{Conflict of Interest: None.}

\section{References}

[Akh95] D. N. Akhiezer, Lie Group Actions in Complex Analysis, Aspects of Mathematics, E27 (Friedr. Vieweg \& Sohn, Braunschweig, 1995). 
[BHPV04] W. P. Barth, K. Hulek, C. A. M. Peters and A. Van de Ven, Compact Complex Surfaces, second edn, Ergebnisse der Mathematik und ihrer Grenzgebiete. 3. Folge. A Series of Modern Surveys in Mathematics [Results in Mathematics and Related Areas. 3rd Series. A Series of Modern Surveys in Mathematics], 4 (Springer, Berlin, 2004).

[Bec78] J. Becker, 'Higher derivations and integral closure', Amer. J. Math. 100(3) (1978), 495-521.

[BW74] D. M. Burns and J. M. Wahl, 'Local contributions to global deformations of surfaces', Invent. Math. 26 (1974), 67-88.

[CDP98] F. Campana, J.-P. Demailly and Th. Peternell, 'The algebraic dimension of compact complex threefolds with vanishing second Betti number', Compos. Math. 112(1) (1998), 77-91.

[Fle88] H. Flenner, 'Extendability of differential forms on non-isolated singularities', Invent. Math. 94(2) (1988), 317-326.

[Gra19] P. Graf, The Lipman-Zariski conjecture in low genus, published electronically in IMRN, July 2019.

[GK14] P. Graf and S. J. Kovács, 'An optimal extension theorem for 1-forms and the LipmanZariski Conjecture', Doc. Math. 19 (2014), 815-830.

[Kod66] K. Kodaira, 'On the structure of compact complex analytic surfaces, II', Amer. J. Math. 88 (1966), 682-721.

[Kol07] J. Kollár, Lectures on Resolution of Singularities, Annals of Mathematics Studies, 166 (Princeton University Press, Princeton, NJ, 2007).

[KM98] J. Kollár and S. Mori, Birational Geometry of Algebraic Varieties, Cambridge Tracts in Mathematics, 134 (Cambridge University Press, Cambridge, 1998).

[Lip65] J. Lipman, 'Free derivation modules on algebraic varieties', Amer. J. Math. 87(4) (1965), 874-898.

[Sei67] A. Seidenberg, 'Differential ideals in rings of finitely generated type', Amer. J. Math. 89 (1967), 22-42.

[vSS85] D. van Straten and J. H. M. Steenbrink, 'Extendability of holomorphic differential forms near isolated hypersurface singularities', Abh. Math. Semin. Univ. Hambg. 55 (1985), 97-110. 